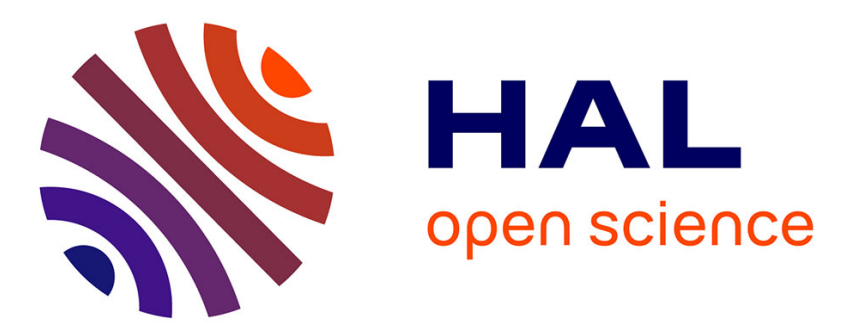

\title{
Développement professionnel et validation des acquis de l'expérience: significations pour les bénéficiaires et les accompagnateurs du dispositif
}

\author{
S. Croity-Belz, Marie-Pierre Cazals-Ferré
}

\section{- To cite this version:}

S. Croity-Belz, Marie-Pierre Cazals-Ferré. Développement professionnel et validation des acquis de l'expérience: significations pour les bénéficiaires et les accompagnateurs du dispositif. Nouvelle revue de psychosociologie, 2016, L'individu pluriel - Enjeux actuels de la construction de soi au travail, 2 (22), pp.111-124. 10.3917/nrp.022.0111 . hal-03224074

\section{HAL Id: hal-03224074 \\ https://hal-univ-tlse2.archives-ouvertes.fr/hal-03224074}

Submitted on 11 May 2021

HAL is a multi-disciplinary open access archive for the deposit and dissemination of scientific research documents, whether they are published or not. The documents may come from teaching and research institutions in France or abroad, or from public or private research centers.
L'archive ouverte pluridisciplinaire HAL, est destinée au dépôt et à la diffusion de documents scientifiques de niveau recherche, publiés ou non, émanant des établissements d'enseignement et de recherche français ou étrangers, des laboratoires publics ou privés. 


\title{
Développement professionnel et validation des acquis de l'expérience : significations pour les bénéficiaires et les accompagnateurs du dispositif
}

\author{
Sandrine Croity-Belz ${ }^{1}$ et Marie-Pierre Cazals-Ferré ${ }^{2}$
}

\section{Introduction}

Les transformations qui ont affecté le monde du travail ces dernières décennies ont dessiné de nouvelles formes de carrière. De plus en plus nomades (Grosjean et Sarnin, 2002), celles-ci sont marquées du sceau de l'incertitude qui, loin de se restreindre à la seule phase de transition école-emploi, s'est immiscée tout au long du parcours jusqu'à s'ériger en norme (Palmade, 2003). Dans ce contexte, le défi majeur des politiques européennes et mondiales en matière d'éducation et d'emploi est de garantir la sécurisation des parcours professionnels (Pinte et Le Squere, 2007). Les actions d'orientation et de formation apparaissent désormais comme des leviers prioritaires pour «prendre en charge les adultes, de plus en plus confrontés à des mobilités en cours de vie active » (Borras et Romani, 2010, p. 14). Elles font par ailleurs écho à des demandes relevant du registre de la gestion des ressources humaines des entreprises pour lesquelles la préoccupation majeure réside dans le rapport le mieux adapté entre le poste de travail et les compétences du futur titulaire du poste (Boutinet, 2009).

Le développement de l'orientation et de la formation tout au long de la vie (OFTLV) devient dès lors un enjeu crucial des politiques publiques et de développement économique. Sont illustratives à cet égard les nombreuses évolutions des cadres législatifs français en matière d'OFTLV. Ainsi, apprendre aux personnes à gérer leur parcours professionnel, rendre le marché du travail plus efficient, accroître la flexibilité par la transférabilité des compétences sont autant d'enjeux qui sous-tendent ces évolutions et qui donnent lieu à des modalités d'OFTLV de plus en plus formalisées autour des logiques d'autoformation et de développement de l'employabilité.

Si l'ambition, avancée par ces orientations, de promouvoir l'égalité des chances ne peut être que louable, elle reste beaucoup plus discutable dès lors qu'elle s'inscrit dans un sillon néolibéral qui prône «l'autonomie de l'individu et sa responsabilité univoque à l'égard de sa propre employabilité » (Le Douaron, 2002, p. 576). On ne peut, en effet, que constater que les individus ont bien intériorisé le glissement vers une nouvelle norme sociale : ce n'est plus à l'entreprise que revient le rôle majeur de garantir l'employabilité de ses salariés, mais aux salariés eux-mêmes de gérer leur carrière en se saisissant des différents dispositifs visant le développement et l'actualisation de leurs compétences. Dans cette perspective, la récente création du Compte personnel d'activité (loi du 27 août 2015 qui rentrera en application en janvier 2017) incite explicitement les individus à prendre en charge eux-mêmes leur parcours professionnel.

De fait, ces évolutions et transformations interrogent... Dans ces injonctions fortes à la responsabilité individuelle adressées aux individus en matière de gestion de carrière, on peut

\footnotetext{
${ }^{1}$ Sandrine Croity-Belz, professeure de psychologie, université Toulouse Jean-Jaurès. scroity@univtlse2.fr

${ }^{2}$ Marie-Pierre Cazals-Ferré, maître de conférences en psychologie, université Toulouse Jean-Jaurès. cazals@univ-tlse2.fr
} 
entrevoir différents risques. Risque pour ceux qui ne sont pas à même, pour diverses raisons, de répondre à ces nouvelles normes, de développer des sentiments d'autoculpabilisation dès lors qu'on leur signifie qu'ils n'ont pas su anticiper, qu'ils n'ont pas su rester compétitifs et employables. Risque donc de renforcer des sentiments de dévalorisation de soi et de nonreconnaissance exacerbés souvent par des conditions de travail (intensification du travail, pressurisation, précarisation...) ou de non-emploi particulièrement éprouvantes. De même, risque pour les individus qui ont les ressources pour répondre à l'injonction d'employabilité d'opter, à l'issue parfois de questionnements, doutes et compromis, pour des orientations professionnelles plus ou moins hétéronormées au détriment de projets professionnels et de vie plus investis au plan personnel.

Sous leur diversité, ces expériences potentiellement aliénantes pour les sujets concernés mobilisent tant les chercheurs qui analysent les processus psychologiques et sociaux à l'œuvre dans les situations de transition professionnelle que les praticiens qui assurent l'accompagnement des personnes confrontées à ces situations. Sur le plan de la recherche, des logiques s'affrontent. Si les conceptions proactives du rapport au travail sont valorisées par des modélisations récentes du développement de carrière (Power et Rothausen, 2003), elles font par ailleurs débat (Dupuy, Le Blanc et Mègemont., 2004 ; Savickas et coll., 2010) dès lors qu'elles sous-estiment d'autres perspectives possibles pour les individus que celles réduites à une logique visant à assurer tant leur maintien au sein du marché de l'emploi que le bon fonctionnement économique de la société. C'est à partir des recherches que nous avons menées sur l'engagement dans des dispositifs d'OTLV (Croity-Belz et Cazals-Ferré. 2010 ; Cazals-Ferré et Croity-Belz, 2009a et 2009b) que nous pouvons prendre part au débat. Nous avons pu montrer que, loin de se réduire à une réponse normative et à de simples logiques de promotion ou de stabilisation professionnelle, l'engagement dans de tels dispositifs mobilise aussi chez les sujets un travail subjectif de construction de sens qui confère à cet engagement des enjeux de personnalisation. Au niveau des pratiques d'accompagnement, ces clivages théoriques ont pour corollaires des positionnements professionnels radicalement différents. Un premier choix de posture peut être, pour le professionnel de l'accompagnement, de s'en tenir à des modalités d'intervention qui visent l'ajustement des individus aux exigences du marché du travail et, dans cette perspective, de les inciter à développer les compétences attendues en matière d'automanagement de carrière. C'est avant tout une logique économique de placement qui prévaut dans ces pratiques.

À distance de ce positionnement, le professionnel peut tenter de se dégager une marge de manœuvre afin de mettre le sujet au centre de sa pratique. Ce choix de posture vise à coconstruire avec la personne accompagnée de nouveaux possibles professionnels dans lesquels elle puisse se reconnaître et être reconnue. Cette posture situe indéniablement l'intervention du consultant du côté du développement de la personne.

Afin d'étayer ces propos, nous allons nous centrer, dans cette contribution, sur un dispositif spécifique, celui de la VAE - qui représente pour nous tant un objet de recherche qu'un domaine d'intervention (en tant que «référentes pédagogiques universitaires » de sujets engagés dans cette démarche) - pour :

- comprendre les logiques d'engagement dans un tel dispositif ;

- dégager comment cette modalité diplômante atypique qui opère un renversement de paradigme dans le champ de l'éducation et de la formation appelle un retour réflexif du sujet sur son histoire de vie qui déborde largement celui requis dans la logique compétence ; 
- questionner les enjeux de personnalisation d'un tel retour réflexif, tant pour la personne qui s'y engage que pour le « référent » qui l'accompagne. Car c'est à travers des processus de négociation et de co-construction du sens de la démarche ainsi que des critères d'évaluation et de validation des «acquis », d'interrogations croisées sur le travail qu'ils mènent ensemble que se jouent les chances de leur développement personnel et professionnel.

\section{La VAE : un dispositif inédit de validation de diplôme}

Si longtemps les seuls apprentissages formels liés à la forme scolaire traditionnelle d'apprentissage étaient reconnus comme support de développement de compétences, les orientations politiques actuelles en faveur de l'emploi (stratégie Europe 2020) accordent une place primordiale à la reconnaissance et à la validation des apprentissages non formels et informels (Doucet, 2015). Il s'agit certes de reconnaître les apprentissages formels, liés à la forme scolaire traditionnelle d'apprentissage, mais aussi ceux - informels - issus «des activités de la vie quotidienne liées au travail, à la famille ou aux loisirs » (Doucet, 2015, p. 11), tout comme ceux - non formels - qui, bien que non inscrits dans un cadre formel, sont présentés «en périodes délimitées avec des objectifs, un programme, des méthodes, des formateurs » (ibid.).

Le dispositif atypique de VAE, mis en place en 2002, permet, par la reconnaissance de ces différents types d'acquis, l'accès à un titre et/ou à un diplôme (Aubret, 2006). La validation du diplôme n'est plus conditionnée par une période de formation, mais par un travail individualisé d'explicitation de la preuve de l'acquisition, via différentes expériences, des compétences correspondantes au diplôme visé. Par l'instauration de ce dispositif, c'est un véritable renversement de paradigme qui s'opère dans le champ de l'éducation et de la formation par le passage d'une logique académique à une logique pragmatique dont l'un des objectifs est d'offrir une nouvelle voie pour faciliter le développement de soi et de ses capacités professionnelles tout au long de la vie (Boutinet, 2009 ; Merle, 2008 ; Presse, 2011).

Si la liberté du temps et de l'espace de travail offerte par ces situations est attrayante, on peut toutefois s'interroger sur les différentes raisons qui amènent les individus à s'engager dans ces nouveaux dispositifs qui bouleversent les modalités traditionnelles de formation et de validation de diplôme. Ainsi, l'engagement dans ces situations traduit-il le seul reflet d'une intériorisation des normes et valeurs d'individualisation et de responsabilisation ? Ne peut-on aussi voir ici la trace d'initiatives du sujet, révélatrices d'une quête identitaire dont les processus d'intersignification (Baubion-Broye, Dupuy et Prêteur, 2013) seraient le moteur ? Les acquis de nos recherches antérieures évoqués ci-après permettent de dégager des pistes de réponses qui invitent à resituer l'engagement en VAE dans la complexité des rapports que le sujet instaure entre ces différents temps et lieux de socialisation.

\section{Engagement en VAE : visée instrumentale ou identitaire ?}

Si certains travaux distinguent les logiques instrumentales d'engagement des logiques identitaires (Personnaz, Quintero et Séchaud, 2005), d'autres (Carré, 1998, 2001 ; Cuvillier, 2004 ; Demailly, 2006) ont l'intérêt de souligner qu'une même logique peut revêtir à la fois des motifs utilitaires et identitaires. Ces derniers n'apparaissent pas d'emblée dans les raisons évoquées par les sujets qui mettent généralement en avant « un rapport au travail verbalisé sur un mode économique » (Crochard, 2007, p 56). Ainsi, si l'engagement dans un dispositif d'OFTLV peut être justifié par une nécessité professionnelle, il ne faut pas négliger pour 
autant de le considérer comme le résultat d'une «décision en tant qu'événement, souvent biographique, de s'engager dans une dynamique inconnue - souvent exigeante en termes de temps, d'implication et complexe en termes de processus décisionnel et d'enjeu identitaire » (ibid., p. 49). L'entrée en VAE s'inscrirait, comme le soulignent Vonthron, Lagabrielle et Pouchard (2007, p. 405) pour la formation continue, «dans une dialectique de choix et de contraintes ».

Les trois types de stratégies d'engagement distinguées par André Demailly (2006) sont illustratives à cet égard. La première, dite stratégie d'alignement, vise à reprendre et à clore un cursus antérieur de formation laissé inachevé. Le projet de valider un diplôme par la VAE peut alors être un moyen pour le sujet de «boucler la boucle ». La deuxième stratégie est celle de l'affrontement. Celle-ci repose sur des arguments plus revendicatifs visant à obtenir en quelque sorte réparation d'une non-reconnaissance de compétences acquises qui attestent pourtant, du point de vue des individus, un niveau de professionnalisme aussi élevé, sinon plus, que celui dont font preuve les titulaires d'un diplôme. La VAE apparaît alors comme un dû de la société qui se doit de reconnaître les compétences développées par les individus plutôt que de les passer sous silence en leur demandant de les acquérir par la voie plus traditionnelle de la formation (Jacques, 2009). Enfin, André Demailly évoque la stratégie d'investissement qui serait plus caractéristique des personnes souhaitant tirer des avantages personnels des possibles ouverts par la VAE tout en y trouvant un levier pour réaliser des projets professionnels qui les valorisent tout autant qu'ils valorisent leur organisation de travail.

Au bilan, le choix de s'engager dans une démarche de VAE n'est pas fortuit. S'il peut relever d'une logique de parcours professionnel, il s'inscrit plus globalement dans une histoire de vie. C'est sans doute ce qui explique que la VAE n'est pas signifiée de façon homogène par les candidats. Pour certains d'entre eux, elle est davantage envisagée comme un instrument «fonctionnel» d'évaluation de leur pratique professionnelle et/ou de bénévolat; pour d'autres, elle prend une dimension supplémentaire en s'inscrivant véritablement dans une démarche biographique qui sous-tend une quête identitaire. Elle s'organise autour de projets toujours signifiants pour chaque candidat, mais différenciés quant à leurs visées et enjeux identitaires.

Ce versant subjectif montre que le choix d'entrer dans ce dispositif ne constitue pas simplement une réponse passive et adaptative aux transformations du monde du travail, mais qu'il a du sens pour le sujet au regard de son histoire et de ses projets de vie. Néanmoins, dans les travaux évoqués, les aspects biographiques restent restreints à la seule sphère professionnelle ou de formation, au détriment d'une prise en compte des activités accomplies et projetées par les sujets dans leurs autres sphères de socialisation. Les raisons de s'inscrire dans un dispositif de VAE semblent ainsi être indépendantes du développement de soi dans les autres registres de vie du sujet. Or, si l'engagement en VAE relève d'un projet professionnel (Lainé, 2004, 2006), il prend aussi son sens au regard de la globalité des activités des individus et, après avoir fait l'objet de délibérations aux niveaux intrapersonnel et interpersonnel, en lien avec les projets de vie et leurs effets escomptés.

\section{Des raisons d'engagement aux ancrages pluriels}

En référence au modèle d'une socialisation active parce que plurielle (Baubion-Broye et Hajjar, 1998 ; Baubion-Broye, Dupuy et Prêteur, 2013 ; Malrieu, 1979), les recherches que nous avons menées (Cazals-Ferré et Croity-Belz, 2009a, 2009b) montrent qu'en situation de 
VAE l'analyse réflexive que les candidats sont amenés à appliquer à leurs expériences les amène inévitablement à interroger les rapports qu'ils instaurent entre leurs différents domaines de vie et ainsi à signifier cet engagement au regard de la pluralité de leurs investissements - passés, présents et projetés. Ce sont ces processus d'intersignification des conduites et d'organisation des relations entre les différents domaines de vie des sujets que nous avons pu approcher dans le cadre d'une recherche qualitative menée auprès de vingt personnes engagées dans une démarche de VAE à l'université (Cazals-Ferré et Croity-Belz, 2009a). Trois types de motifs d'engagement en VAE se sont dégagés de nos analyses ${ }^{3}$ et permettent d'illustrer qu'un tel engagement est lourd d'enjeux tant professionnels que personnels pour l'individu et est loin d'être un événement circonscrit à la seule sphère professionnelle.

Nous avons ainsi pu repérer une première logique de promotion professionnelle non exempte pour autant de conflits aux niveaux intrapersonnel et interpersonnel. Les sujets qui se reconnaissent dans cette logique, bien que manifestant une forte valorisation de leur sphère professionnelle, tendent à privilégier les activités relevant des domaines hors travail (activités familiales, amicales, de loisirs). Ils font part d'un débordement parfois envahissant des contraintes professionnelles sur les autres sphères d'investissement («Quand on a la pression au boulot, ça se répercute sur la vie privée »). La stratégie identitaire dominante ici, l'affrontement, redouble au niveau interpersonnel et positionnel (là où il y a un fort enjeu de reconnaissance) cette dynamique conflictuelle. L'engagement en VAE, perçu comme un moyen nécessaire pour atteindre un projet d'ascension et de reconnaissance professionnelle, n'est ainsi pas sans interférence avec les engagements du sujet dans ses autres sphères de vie.

La deuxième logique, dite de stabilisation, est représentée par des individus qui valorisent fortement leur sphère professionnelle relativement aux autres domaines de vie. Toutefois, la dimension conflictuelle entre domaines de vie n'apparaît pas. Les individus expriment des inquiétudes quant à leur devenir professionnel et à l'évolution du marché du travail. Ils font part d'un regret d'avoir interrompu trop tôt leurs études. En faisant le bilan de leurs expériences professionnelles, ces sujets se rendent compte aussi que certains acquis professionnels (capacités de négociation, patience...) leur sont utiles dans leur vie hors travail. La VAE apparait alors comme un levier pour atteindre une stabilisation professionnelle référée ici à une visée d'unification de soi tant au plan de l'histoire de vie («boucler la boucle ») qu'au niveau des rapports entre les différents domaines d'activités (en activant les échanges de ressources entre domaines de vie). Cette dynamique d'unification de soi semble s'opérer autour de la sphère professionnelle perçue d'une part comme potentiellement réparatrice d'une trajectoire non satisfaisante et, d'autre part, comme support dans les ressources qu'elle peut apporter aux autres sphères d'investissement.

La dernière logique, celle de l'équilibre, est représentée par des personnes qui recherchent avant tout l'épanouissement dans leur travail, qu'elles expriment en termes de plaisir, voire bonheur : «... faire son travail avec plaisir», " pouvoir apporter tout ce qu'on sait à son travail et ses collègues », «être heureux d'aller travailler tous les jours ». Pour elles, la VAE constitue un moyen d'accéder à cet épanouissement. Au-delà d'un idéal professionnel, l'épanouissement est également recherché par le maintien d'un équilibre entre le domaine du travail et les autres investissements tels que la famille, les loisirs, la santé, les amis. C'est parce qu'ils refusent de hiérarchiser leurs investissements que ces sujets organisent leur

\footnotetext{
${ }^{3}$ Les données ont été traitées à partir d'une analyse lexicale et morphosyntaxique à l'aide du logiciel d'analyse des données textuelles Alceste (Reinert, 1993)
} 
système des activités sur le mode du cloisonnement. Le fait de cloisonner les échanges entre les différentes sphères d'activités semble être garant de cet équilibre ( $\mathrm{Il}$ ne faut pas que le travail déborde sur ma vie privée »; «Je ne peux pas me passer de mon travail, mais il faut aussi exister dans le privé », "J'ai une autre vie très enrichissante et je ne mélange pas les espaces, il me faut les deux »).

Les trois logiques que nous avons dégagées dessinent chacune une combinaison originale de buts à court terme, de mobiles plus identitaires ancrés et construits dans l'histoire de vie des sujets ainsi que des modes d'échanges que les sujets instaurent entre leurs différents domaines de vie. Ainsi, les raisons d'engagement en VAE ne peuvent être réduites à une réponse ajustée aux incitations politiques axées sur la sécurisation des parcours professionnels. Elles trouvent leurs origines dans les contradictions, doutes, ambivalences, craintes liées à la rencontre des bouleversements qui affectent le monde du travail avec les rapports à soi et aux autrui significatifs que le sujet construit et développe en ses différents temps et lieux de socialisation. L'engagement dans des situations d'OFTLV telles que la VAE témoignerait alors d'une «quête personnelle, à maints égards solitaire, et fondamentalement sociale » (Baubion-Broye et Malrieu, 1987). Les logiques sous-jacentes à cet engagement ne se restreignent pas à des finalités instrumentales inhérentes à l'injonction contemporaine de rester employable. Sont à l'œuvre dans cet engagement des dynamiques identitaires qui reposent sur l'articulation de différentes temporalités, passé, présent et avenir, et d'investissements multiples dans des registres de vie différents. Cet engagement ne relève pas du seul registre professionnel mais est intégré dans une histoire de vie, articulé à des projets de vie, soutenu par une représentation de soi et de ses compétences construites au cours de diverses expériences (Dupuy, 1998). Il trouve ancrage dans des insatisfactions ou crises personnelles qui ont jalonné la trajectoire de vie et dont Malrieu (1979, 2003), dans son modèle d'une socialisation plurielle, dit qu'elles sont le moteur possible de leur dépassement.

\section{Réflexivité et co-construction : voies de personnalisation ?}

Les différents éléments évoqués soulignent à quel point l'engagement en VAE peut être lourd d'enjeux tant professionnels que personnels (Lainé, 2004). Or, la réussite ne va pas de soi. L'extrait d'entretien suivant est éloquent à cet égard : «Cette démarche. ça m'apporte du tracas, une épine dans le pied, un caillou dans la chaussette... le foutoir d'aller chercher toutes ces choses paumées » (Cazals-Ferré et Croity-Belz, 2010). Si la démarche de VAE peut agir comme un « révélateur» psychologique et devenir un véritable enjeu pour le bien-être de certains candidats, elle peut, à l'inverse, enfermer certains autres dans des logiques aliénantes (Cazals-Ferré et Croity-Belz, 2009a). Le travail de retour sur soi et sur ses activités qu'elle mobilise met à l'épreuve la représentation que les sujets ont d'eux-mêmes et de leurs compétences (Gourmelen, 2006 ; Jacques, 2009 ; Paul et Clavier, 2007 ; Rémery, 2012). Il n'est pas non plus exempt de débordement de la sphère de formation sur les activités développées par les sujets dans leurs autres domaines de vie. Ces effets probables ont pour corollaire un risque de découragement, voire d'abandon (Besson, 2008 ; Blay et Jouin, 2006), accrus notamment par l'isolement induit par ces nouvelles modalités d'individualisation des parcours de formation.

Accompagner le bénéficiaire tout au long de sa démarche de professionnalisation est alors une condition primordiale. L'accompagnement permet d'accroître les chances de validation totale (Bonaïti, 2008) en réduisant la complexité du travail attendu dans le dossier de preuve et en favorisant la mobilisation des candidats dans le travail de rédaction attendu (Havet, 2014). 
Toutefois, ce n'est pas tant cette aide motivationnelle et rédactionnelle qui est le ressort possible d'un développement personnel et professionnel que le travail subjectif de retour historique sur soi favorisé par l'accompagnement. Cette réflexivité est mise au travail par l'accompagnateur et peut prendre la forme de dialogues au sens de Malrieu (2003). Dialogues avec soi au travers desquels se rencontrent et se confrontent les engagements du sujet (ses choix et initiatives personnels au regard de ses pluralités d'appartenance et de son histoire de vie) ; ses diverses activités - passées, présentes et projetées - marquées de ses relations à soi (images de soi), à autrui (au travers des processus d'identification et de contre-identification, de comparaison sociale) et aux groupes sociaux d'appartenance (représentation des normes et des relations entre ces différents groupes). Ces dialogues révèlent qu' «à côté des engagements acceptés et même voulus il en est d'autres qui posent des questions parfois dramatiques au sujet » (Malrieu, 2003, p. 58) et qui, en cela, peuvent être générateurs de conflits. Ces divergences, contradictions - plus ou moins éprouvantes pour le sujet -entre les sollicitations sociales liées à la pluralité de ses ancrages sociaux et les représentations qu'il a de lui-même et de ses relations aux autres, sont objectivées via l'activité réflexive soutenue par autrui qui ouvre ainsi pour le sujet la voie d'un dégagement possible de ces incohérences par la personnalisation.

Ces éléments montrent la nécessité d'appréhender l'accompagnement comme une «situation de médiation propice à l'engagement du travail réflexif » (Cuvillier, 2004, p. 129) et invitent à s'interroger sur le rôle spécifique que joue l'accompagnateur dans le dépassement des «tensions identitaires » perçues par les candidats (Rémery, 2012). Une des finalités de l'accompagnement n'est alors pas tant d'aider les sujets à s'adapter aux changements auxquels ils sont confrontés que de les aider à objectiver et à dépasser les contradictions qu'ils perçoivent dans leur confrontation à ces changements. L'objectivation contribue au déplacement du subi - source potentielle d'aliénation - vers le pouvoir d'agir, lequel permet au sujet de se (re)penser comme capable de définir de nouveaux possibles, capable de transformer un tant soit peu ses environnements pour davantage s'y reconnaître et se reconnaître. Ce travail subjectif de dépassement des contradictions vécues ne peut se faire sans la médiation d'autrui.

\section{La reconnaissance institutionnelle de l'accompagnement}

Est rendue ici saillante la nécessité « d'organiser le fonctionnement institutionnel (institutions de formation et organisation de travail) pour que l'expérience puisse être reconnue comme valeur» (Aubret, 2006, p. 385). Il revient donc aux organismes qui la mettent en place de reconnaître que l'aboutissement d'une VAE n'est pas de façon linéaire liée à la responsabilité d'un candidat, mais se joue également dans la somme des représentations, investissements, fruits de l'expérience, codes et normes implicites des principaux protagonistes - candidats, accompagnateurs, membres du jury (Auras, 2014). L'institution peut aussi avoir sa part de responsabilité via les modalités de professionnalisation des accompagnateurs qu'elle met en œuvre.

Au sein des universités, la mise en place de la VAE a conduit à confier aux enseignants chercheurs le rôle de « référent pédagogique » pour accompagner les candidats. Leur rôle est crucial, car ils doivent aider les candidats à faire leurs les règles du jeu universitaire lors d'un parcours de formation atypique de quelques semaines (Auras, 2014). Mais tout ceci n'est pas écrit ou dit explicitement à ces référents. Si la réalisation d'une VAE est loin d'être évidente pour les candidats, l'accompagnement pédagogique est également jalonné, pour les référents, d'ambiguïtés et d'incertitudes. Il n'existe pas à proprement parler de formation à 
l'accompagnement. Aussi, les postures de formateur, d'accompagnateur, d'évaluateur sontelles sujettes à de nombreuses variations (Crognier, 2010), tant dans les pratiques que dans les représentations. Dans une étude qualitative exploratoire menée auprès de huit enseignantsréférents en VAE à l'université (Croity-Belz et Cazals-Ferré, 2010), nous avons-nous-mêmes pu repérer deux principales modalités d'appropriation du rôle de référent pédagogique. Si pour certains, l'accompagnement s'assimile avant tout à une posture pédagogique, pour d'autres, il suppose nécessairement une distanciation à l'égard de cette posture (« aide un peu à la réflexivité », «aide un peu à l'explicitation, à l'analyse de l'expérience », «mon accompagnement, je le plaçais dans : cheminer avec quelqu'un »).

Le manque perçu d'accompagnement dans l'exercice de ce nouveau rôle amène les référents à se professionnaliser par autoformation au fur et à mesure de leur expérience de référent («Je suis allée chercher des choses mais de façon autonome », «je me suis débrouillée » ou encore «au fil du temps on se construit une petite expertise »). Toutefois, si les référents œuvrent « en aveugle » tout en mobilisant leurs acquis issus de leur expérience d'enseignant, ils ne souhaitent pas pour autant que les modalités d'accompagnement soient standardisées. De tels éléments mettent en exergue que la posture d'accompagnateur ne va pas de soi, ne s'inscrit pas nécessairement dans l'ordre des choses et semble loin d'être homogène (Crognier, 2010). En outre, le risque lié à une non-reconnaissance de la spécificité de la mission d'accompagnement est non seulement l'interruption de la démarche VAE par les candidats, mais aussi le désengagement des enseignants chercheurs de cette mission. Toutefois, la reconnaissance ne signifie pas de tendre vers une homogénéisation des pratiques, mais de coconstruire avec les acteurs concernés les conditions d'un accompagnement potentiellement vecteur de développement personnel et professionnel tant pour le référent que pour le bénéficiaire.

\section{Conclusion}

L'exemple des situations de VAE a bien montré que, sous l'impulsion des orientations politiques des pays membres de la Commission européenne, les changements actuels qui caractérisent les contextes de travail et de formation participent à la construction des conduites individuelles. Pour autant, en référence au modèle d'une socialisation plurielle et active qui oriente nos travaux, cette construction ne doit pas être appréhendée comme le jeu d'une simple détermination sociale des conduites, mais comme résultant d'une interstructuration du sujet et des facteurs sociaux et sociétaux (normes, valeurs, émergence de nouveaux dispositifs de formation...). Dans cette contribution, nous nous sommes particulièrement efforcées de rendre compte des processus psychosociaux sous-jacents à l'appropriation, par les sujets, de la norme croissante de l'individualisation des parcours professionnels et de formation promue comme un levier pour atteindre « une croissance économique durable avec des emplois meilleurs et plus nombreux et une plus grande cohésion sociale »(Pinte, 2012, p. 246). Nous avons ainsi repéré que, loin d'être assujettis aux transformations sociétales, les sujets acceptent, à des degrés différents, de promouvoir ces changements en s'inscrivant dans les dispositifs atypiques qui en découlent, mais sous la condition qu'ils puissent les faire leurs au regard des rapports à soi et à autrui qu'ils construisent au cours de leur histoire et de leurs engagements pluriels.

La finalité étant pour les sujets accompagnés d'atteindre une possible personnalisation dont l'élaboration d'œuvres qui portent leur marque - telles que les traces écrites de l'explicitation des acquis d'expériences - peut être le support. Autrement dit, l'accompagnement à la VAE ne renvoie pas à une situation pédagogique traditionnelle où il peut être question de transmettre un contenu disciplinaire arrêté et fixé par des instructions officielles. La 
complexité de la tâche réside en ce que l'accompagnateur ne peut savoir a priori ce que le processus d'analyse réflexive des pratiques va générer comme effet chez le candidat aux prises avec la VAE et avec son histoire personnelle et professionnelle (Crognier, 2010).

\section{Bibliographie}

Aubret, J. 2006. «Comment évaluer et valider les acquis de l'expérience ? », dans C. Lévy-Leboyer, C. Louche, J.-P. Rolland (sous la direction de), RH : les apports de la psychologie du travail. Management des personnes, Paris, Les Éditions d'organisation, 373- 392.

Auras, E. 2014. «Le travail des jurys de VAE ou la mise en scène de l'expérience », Formation emploi, 122, 2, 51-67 [en ligne], www.cairn.info/revue-formation-emploi-2013-2-page-51.htm.

Baubion-Broye, A. ; Malrieu, P. 1987. "Pour une étude de la structure et de la genèse du projet », dans M. Bru et L. Not (sous la direction de), Où va la pédagogie du projet ?, Toulouse, Éditions universitaires du Sud, 129-150.

Baubion-Broye, A. ; Hajjar, V. 1998. «Transitions psychosociales et activités de personnalisation », dans A. Baubion-Broye (sous la direction de), Evénements de vie, transitions et construction de la personne, Toulouse : Eres, 17-43.

Baubion-Broye, A. ; Dupuy, R. ; Prêteur, Y. 2013. Penser la socialisation en psychologie. Actualité de l'œuvre de Philippe Malrieu. Toulouse, érès.

Besson, E. 2008. «Valoriser l'acquis de l'expérience : une évaluation du dispositif de VAE », Rapport ministériel du secrétariat d'État chargé de la prospective, de l'évaluation des politiques publiques et $d u$ développement de l'économie numérique, [en ligne], www.ladocumentationfrancaise.fr/var/storage/rapports-publics/084000590.pdf.

Blay, X. ; Jouin, C. 2006. Enquête auprès des candidats à la validation des acquis de l'expérience : perception du processus, nature des effets, causes de l'abandon ou de l'interruption de la démarche, Nantes, CAFOC.

Bonaïti, C. 2008. «Le parcours des candidats à la validation par les acquis de l'expérience des titres et diplômes de niveau V : mieux vaut être accompagné et diplômé pour réussir », DARES, 34, 2, 1-7.

Borras, I. ; Romani, C. 2010. « Orientation et politiques publiques », Formation emploi, 109, 9-22.

Boutinet, J.-P. (sous la direction de). 2009. L’ABC de la VAE, Toulouse, érès.

Carré, P. 1998. «Motifs et dynamiques d'engagement en formation », Éducation permanente, 136, 119-131.

Carré, P. 2001. De la motivation à la formation, Paris, L'Harmattan.

Cazals-Ferré, M.-P. ; Croity-Belz, S. 2009a. «Étude exploratoire de l'engagement dans un dispositif de validation des acquis de l'expérience (VAE) : le rôle des valorisations et des échanges inhérents aux différents domaines de vie », Pratiques psychologiques, 15, 2, 239-254.

Cazals-Ferré M.-P.; Croity-Belz, S. 2009b. «Maintien ou désengagement des candidats en validation des acquis de l'expérience : le rôle des soutiens sociaux », $51^{e}$ congrès annuel de la Société uychologie (SFP) : psychologie et enjeux de société, Toulouse, 17-19 juin.

Cazals-Ferré, M.-.P ; Croity-Belz, S. 2010. «Quels types de difficultés inhérentes au parcours de la VAE sont impliqués dans le maintien vs l'interruption de ce parcours ? Le rôle modérateur des stratégies d'activation des ressources interpersonnelles issues des différents domaines de vie des candidats », Actes du $16^{e}$ congrès de l'Association internationale de psychologie du travail de langue française (AIPTLF), 6-9 juillet.

Croity-Belz, S. ; Cazals-Ferré, M.-P. 2010. «Accompagnement et validation des acquis de l'expérience à l'université », Colloque international CNAM-INETOP, Paris, 17-19 mars.

Crochard, M. 2007. «Contribution à l'étude des liens entre engagement en formation et rapport au travail », Savoirs, hors-série, 4, 49-60. $22,132-152$.

Crognier, P. 2010. «Accompagner les candidats à la VAE. Le mythe de la juste distance », Savoirs, 140.

Cuvillier, B. 2004. «La Vae, un tremplin pour la formation », Éducation permanente », 158, 127-

Demailly, A. 2006. «La validation des acquis de l'expérience (VAE), une révolution silencieuse. Enjeux individuels et collectifs de la validation des acquis de l'expérience », [en ligne] archive.mcxapc.org/docs/ateliers/0606demailly.pdf.

Doucet, S. 2015. «Rapport d'information déposé par la commission des affaires européennes sur la validation de l'apprentissage non formel et informel », [en ligne] www.assembleenationale.fr/14/pdf/europe/rap-info/i2926.pdf. 
Dupuy, R. 1998. «Transitions et transformation des identités professionnelles : le cas des adultes en situations de formation continue », dans A. Baubion-Broye (sous la direction de). Événements de vie, transitions et construction de la personne, Toulouse, érès, 44-71.

Dupuy, R.; Le Blanc, A.; Mègemont, J.-L. 2004. «Incertitudes au cours de la carrière et construction des perspectives temporelles », Psychologie du travail et des organisations, 12, 167-183.

Gourmelen, B. 2006. La validation des acquis de l'expérience, Paris, L'Harmattan.

Grosjean, M. ; Sarnin, P. 2002. «Les parcours professionnels », Éducation permanente, 150, 9-22.

Havet, N. 2014. «Le rôle de l'accompagnement dans la réussite des parcours de validation des acquis de l'expérience », Formation-Emploi, 125, 47-68.

Jacques, M.H. 2009. «Validation des acquis de l'expérience et accompagnement : seconde chance ou nouveau risque d'inégalités ? », L'orientation scolaire et professionnelle, 38, 2,161-181.

Lainé, A. 2004. « D’une expérience à l'autre », Éducation permanente, 159, 2, 23-36.

Lainé, A. 2006. VAE, quand l'expérience se fait savoir; L'accompagnement en validation des acquis, Toulouse, érès.

Le Douaron, P. 2002. «La formation tout au long de la vie. Promotion René Cassin », Revue française d'administration publique, 104, 4, 573-580.

Malrieu, P. 1979. «La crise de personnalisation. Ses sources et ses conséquences sociales », Psychologie et éducation, 3, 3, 1-18.

Malrieu, P. 2003. La construction du sens dans les dires autobiographiques, Toulouse, érès.

Merle, 2008. "Groupe de travail sur la validation des acquis de l'expérience », [en ligne] www.ladocumentationfrancaise.fr/var/storage/rapports-publics/094000020.pdf.

Palmade, J. (sous la direction de). 2003. L'incertitude comme norme, Paris, Puf.

Paul, M. ; Clavier, L. 2007. «Le statut de la parole en validation des acquis de l'expérience», Recherches en éducation, 2, 68-80.

Personnaz, E. ; Quintero, N. ; Séchaud, F. 2005. «Parcours de VAE, des itinéraires complexes, longs, à l'issue incertaine », Céreq, Bref, 224.

Pinte, G. ; Le Squère, R. 2007. «De la flexibilité à la sécurisation des parcours professionnels : l'outil VAE et les intérimaires », Savoirs, 4, (Hors série), 81-93.

Pinte, G. 2012. "La longue marche de la reconnaissance et de la validation des acquis de l'expérience (RVAE) dans le champ de l'éducation et de la formation », Carriérologie, 12, 3, 243-256.

Power, S.J. ; Rothausen, T.J. 2003. «The work-oriented mid-career development model: An extension of Super's maintenance stage », The Counselling Psychologist, 31, 2, 157-197.

Presse, M.-C. 2011. «De la validation des acquis à la professionnalisation des formateurs », Éducation permanente, 188, 3, 37-52.

Reinert, M. 1993. «Les mondes lexicaux et leur logique à travers l'analyse statistique d'un corpus de récits de cauchemars », Langage et Société, 60, 5-39.

RémEry, V. 2012. «La VAE, une situation d'action dans la gestion de carrière », Carriérologie, 12, 3, 257-269.

Savickas, M.-L. ; Nota, L. ; Rossier, J. ; Dauwalder, J.-P. ; Duarte, M.E. ; Guichard, J. ; Soresi, S. ; Van Esbroeck, R.; Van Vianen, A.E.M.; Bigeon, C. 2010. «Construire sa vie (Life designing): un paradigme pour l'orientation au XXI siècle », L'orientation scolaire et professionnelle, 39, 1, 5-39.

Vonthron, A.M. ; Lagabrielle, C. ; Pouchard, D. 2007. «Le maintien en formation professionnelle qualifiante: effets de déterminants motivationnels, cognitifs et sociaux », L'orientation scolaire et professionnelle, 36, 3, 401-420.

\section{Sandrine Croity-Belz et Marie-Pierre Cazals-Ferré, Développement professionnel et validation des acquis de l'expérience: significations pour les bénéficiaires et les accompagnateurs du dispositif}

\section{Résumé}

Cet article a pour objectif d'examiner les incidences sur les individus des injonctions actuelles en matière de responsabilité de sa carrière. Au niveau de la recherche, il s'agit de montrer, au regard du modèle d'une socialisation active parce que plurielle, que l'engagement dans une démarche de validation des acquis de l'expérience ne relève pas du seul registre professionnel, mais est à comprendre dans la complexité des rapports que le sujet instaure entre ces différents temps et lieux de socialisation. Au niveau des pratiques, ceci conduit à reconnaître l'importance du référent VAE dans l'accompagnement du travail sur soi et dans la co-construction du sens de la démarche. 


\title{
Mots-clés
}

Validation des acquis de l'expérience, orientation et formation professionnelles, engagement, employabilité, développement de soi, personnalisation

\section{Sandrine Croity-Belz and Marie-Pierre Cazals-Ferré,Validation of prior experience : professional developmentfor individuals and educational referents}

\begin{abstract}
This article aims to examine the impact on individuals of current orders for accountability of his career. At the research level, it is to show, in respect of an active and plural model of socialization, that engagement in a process of validation of prior experience falls not only the professional field but is to understand in relation with the complex relationships that individual develops between these different times and places of socialization. In practices, this leads to recognize the importance of the pedagogic referent in the accompaniment of reflexivity and the co-construction of meaning in the process.
\end{abstract}

\section{Keywords}

Validation of the prior experience, vocational guidance and training, commitment, employability, self development, personalization. 